\title{
BMJ Open Characteristics and outcomes of women using emergency medical services for third-trimester pregnancy-related problems in India: a prospective observational study
}

Matthew C Strehlow, ${ }^{1}$ Jennifer A Newberry, ${ }^{1}$ Corey B Bills, ${ }^{2}$ Hyeyoun (Elise) Min, ${ }^{3}$ Ann E Evensen, ${ }^{4}$ Lawrence Leeman, ${ }^{5}$ Elizabeth A Pirrotta, ${ }^{1} \mathrm{G}$ V Ramana Rao, ${ }^{6}$ $S \vee$ Mahadevan $^{1}$

To cite: Strehlow MC, Newberry JA, Bills CB, et al. Characteristics and outcomes of women using emergency medical services for thirdtrimester pregnancy-related problems in India: a prospective observational study. BMJ Open 2016;6: e011459. doi:10.1136/ bmjopen-2016-011459

- Prepublication history and additional material is available. To view please visit the journal (http://dx.doi.org/ 10.1136/bmjopen-2016011459).

Received 22 February 2016 Revised 2 May 2016 Accepted 26 May 2016

CrossMark

For numbered affiliations see end of article.

Correspondence to Dr Matthew C Strehlow; strehlow@stanford.edu

\section{ABSTRACT}

Objectives: Characterise the demographics, management and outcomes of obstetric patients transported by emergency medical services (EMS).

Design: Prospective observational study.

Setting: Five Indian states using a centralised EMS agency that transported 3.1 million pregnant women in 2014.

Participants: This study enrolled a convenience sample of 1684 women in third trimester of pregnancy calling with a 'pregnancy-related' problem for free-ofcharge ambulance transport. Calls were deemed 'pregnancy related' if categorised by EMS dispatchers as 'pregnancy', 'childbirth', 'miscarriage' or 'labour pains'. Interfacility transfers, patients absent on ambulance arrival and patients refusing care were excluded.

Main outcome measures: Emergency medical technician (EMT) interventions, method of delivery and death.

Results: The median age enrolled was 23 years (IQR 21-25). Women were primarily from rural or tribal areas $(1550 / 1684(92.0 \%))$ and lower economic strata $(1177 / 1684(69.9 \%))$. Time from initial call to hospital arrival was longer for rural/tribal compared with urban patients (66 min (IQR 51-84) vs 56 min (IQR 42-73), respectively, $p<0.0001$ ). EMTs assisted delivery in 44 women, delivering the placenta in $33 / 44(75 \%)$, performing transabdominal uterine massage in 29/33 $(87.9 \%)$ and administering oxytocin in none $(0 \%)$. There were 1411 recorded deliveries. Most women delivered at a hospital (1212/1411 (85.9\%)), however $126 / 1411(8.9 \%)$ delivered at home following hospital discharge. Follow-up rates at 48 hours, 7 days and 42 days were $95.0 \%, 94.4 \%$ and $94.1 \%$, respectively. Four women died, all within 48 hours. The caesarean section rate was $8.2 \%(116 / 1411)$. On multivariate regression analysis, women transported to private hospitals versus government primary health centres were less likely to deliver by caesarean section $(\mathrm{OR}$ $0.14(0.05-0.43))$

\section{Strengths and limitations of this study}

This study is a novel, prospective assessment of obstetric patients calling for emergency medical services across five states in India.

- Data were collected real time, and 42-day follow-up rates were excellent (94.1\%).

- Generalisability may be limited as it was a convenience sample during daytime hours.

- Limited data on in-hospital management were collected.

Conclusions: Pregnant women from vulnerable Indian populations use free-of-charge EMS for impending delivery, making it integral to the healthcare system. Future research and health system planning should focus on strengthening and expanding EMS as a component of emergency obstetric and newborn care (EmONC).

\section{INTRODUCTION}

As the global health community's priorities transition from the United Nation's Millennium Development Goals (MDGs) to the Sustainable Development Goals (SDGs), improving access to quality maternal care remains a top priority. ${ }^{1}{ }^{2}$ SDG 3.1 specifies a new global maternal mortality ratio (MMR) target of $<70$ per 100000 live births by 2030 . To accomplish this goal, the WHO's Global Strategy for Women's, Children's, and Adolescents' Health (2016-2030) identifies facility-based childbirth with a skilled birth attendant and comprehensive emergency obstetric care as essential, evidenced-based interventions. The impact of these interventions, however, is critically limited by inequities in access to care. ${ }^{3}{ }^{4}$ A significant limit to 
access is timely transport, which may be affected by distance, ${ }^{5}$ cost $^{6}$ and even social networks. ${ }^{7}$ Yet to date, programmes aimed at improving access by decreasing barriers to transport often fail to reach the most vulnerable populations and have been unable to demonstrate a consistent reduction in maternal deaths. ${ }^{8}$

In India, the country-wide MMR was 174 in 2015, and is highly variable by state and urbanisation. ${ }^{9}{ }^{10}$ Public health efforts have aimed to reduce this high MMR through a number of interventions yet few have addressed the second delay, the time to reach care. Janani Express Yojana (JEY) transport programme was created to improve access to timely obstetric care. To do so, JEY worked with the Janani Suraksha Yojana (JSY) programme, which incentivises women by providing conditional cash transfers to deliver at facilities. In the state of Madhya Pradesh, the JEY programme achieved moderate penetration with $35 \%$ of pregnant patients using their transport services. However, patients encountered frequent delays when transported by JEY vehicles. ${ }^{11}$ Their 2-hour average transport time was comparable to patients that used public transport, with over $50 \%$ of patients taking longer than 4 hours to arrive at a facility. ${ }^{11}$ GVK Emergency Management and Research Institute (GVK EMRI) is a public-private partnership that also provides free ambulance transport along with prehospital stabilisation care, and can be easily accessed using a toll free phone number (108). In some states, they provide a separate parallel service (102) for routine pregnancy-related transport, including delivery. Call management, dispatch and online medical direction are provided by a centralised, state-level, emergency call centre that supports a fleet of ambulances, strategically distributed to optimise response times. Obstetric emergencies are the most common reason to call GVK EMRI for assistance, with an estimated 3.1 million transports for pregnancy-related problems in 2014. ${ }^{12}$

Despite the extensive use of ambulance transport services for obstetric indications in India, the critical role of prehospital care providers in managing obstetric patients often goes under-recognised by national and international agencies. For example, prehospital care providers are not mentioned in the Every Women Every Child Global Strategy 2.0. ${ }^{3}$ Emergency medical services (EMS) systems and prehospital care providers have the potential to significantly improve the outcomes of obstetric patients through timely prehospital medical interventions and transport to facility-based care. ${ }^{13}$ Yet, to date, limited research exists describing their obstetric patients, the care provided, or patient outcomes. ${ }^{14} 15$ Our study seeks to characterise the demographics, management and outcomes of third-trimester obstetric patients transported and treated by GVK EMRI.

\section{METHODS}

We conducted a prospective observational study of patients calling 108 for pregnancy-related problems across five states in India-Andhra Pradesh, Assam, Gujarat, Karnataka and Meghalaya. Launched in 2005, GVK EMRI covers the entirety of 17 states and union territories, providing free-of-charge ambulance transport and emergency care to over 750 million people in India (figure 1). The vast majority of ambulances are staffed by a driver and a single emergency medical technician (EMT). Ambulances transport all types of emergency patients and EMTs are trained to provide basic adult and paediatric emergency care in addition to emergency obstetric care and resuscitation. They are empowered to administer life-saving medications such as oxytocin and magnesium, under the oversight of real-time, physicianguided medical direction and via standard care protocols (see online supplementary material 1). Following initial assessment and treatment, ambulances transport patients to the nearest hospital, unless otherwise requested by the patient or her family.

We enrolled a convenience sample of patients for a defined 6-week period from 17 February to 10 April 2014. Patients were enrolled Monday through Saturday, during daytime hours for 6 hours per day. Any woman in her third trimester of pregnancy who called 108 for a pregnancy-related problem was eligible for enrolment. A call was considered 'pregnancy-related' if it was categorised by the EMS dispatch officer as a call for 'pregnancy', 'childbirth', 'miscarriage', or 'labour pains'. Exclusion criteria included calls for interfacility transfers, patients who were absent on EMT arrival and patients who refused care services. At initial enrolment, trained research assistants used a standardised questionnaire to collect data in real time by phone from the EMTs caring for patients. Data included patient demographics, prior and current obstetric history, and physical exam findings. After EMTs completed patient transport, research assistants recontacted EMTs by phone to collect additional information such as EMT interventions at the scene and en route. At the time of initial enrolment, two phone numbers were obtained, the patient's and a friend's or relative's, to limit the number lost in follow-up.

Patients who did not deliver prior to hospital arrival or en route were followed up by phone at 48 hours and 7 days. If they did not deliver by 7 days, they were excluded from further analysis. All patients who delivered, prior to EMT arrival through 7 days after the dispatch call, were followed up by phone at 48 hours, 7 days and 42 days postpartum.

The study's primary outcomes were caesarean section and death. Demographics, obstetric history, current signs and symptoms, transport distances and times, and care characteristics were compared using $\chi^{2}$ analysis for categorical variables (or Fisher's exact test when appropriate) and Wilcoxon two-sample test for continuous variables to identify univariate predictors of caesarean section. Multivariate logistic regression analysis was used to determine predictors of caesarean section based on significance in the univariate analysis. A $p<0.05$ was 


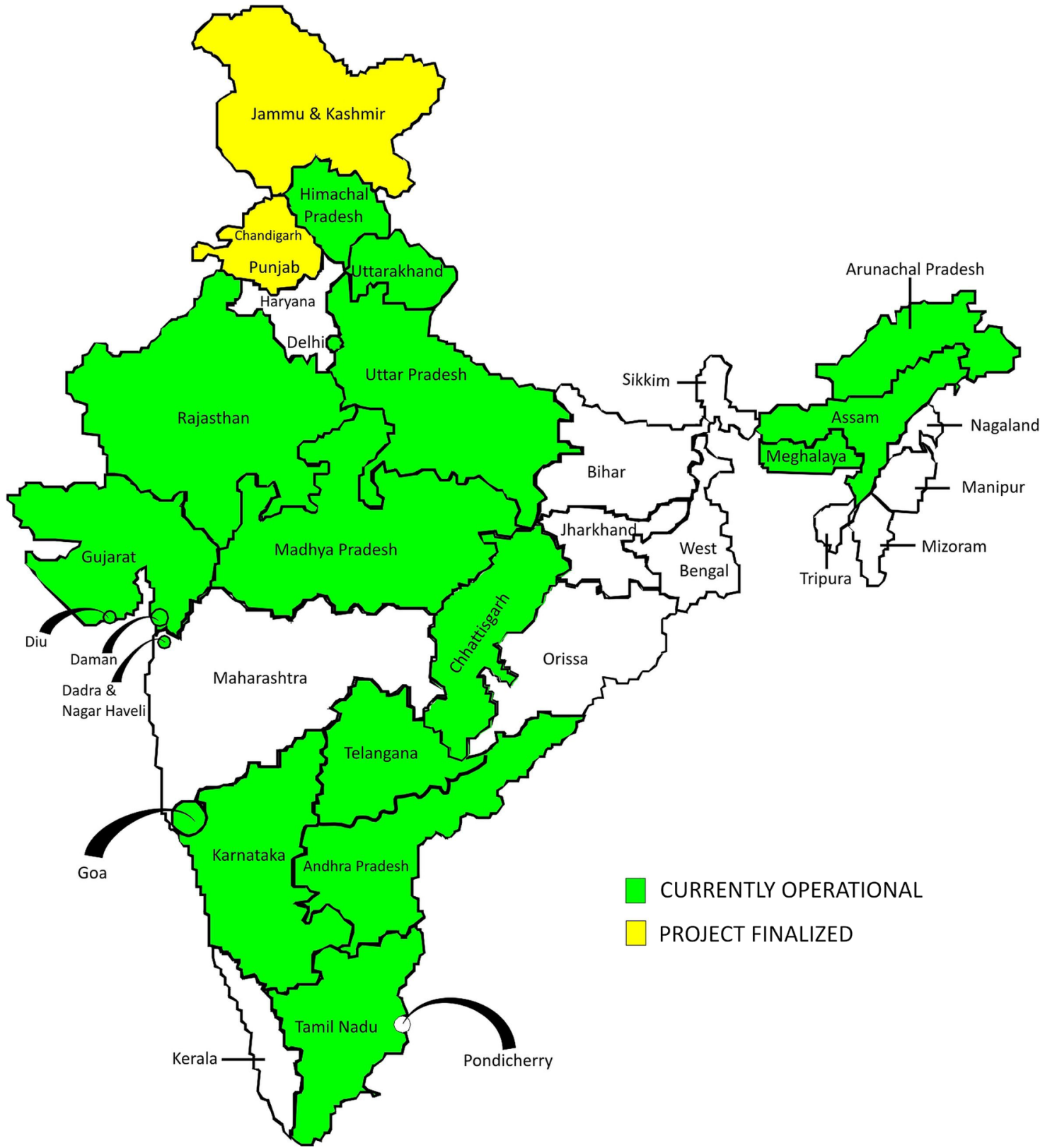

Figure 1 Map of India showing location of GVK EMRI emergency medical services.

considered significant. All data analysis was conducted via SAS Enterprise Guide for Windows, V.4.3 (SAS Institute Inc., Cary, USA). ORs and 95\% CIs are reported for all model variables. Per GVK EMRI's standard operating procedures participants provided verbal consent for care, transport, data collection and follow-up at the time of enrolment. The study was approved by the Institutional Review Board at Stanford University (IRB\#18185) and the Ethics and Research Committee at GVK EMRI. The study was funded jointly by Stanford University and GVK EMRI.

\section{RESULTS}

We enrolled 1684 women, $~ 1.7 \%$ of all pregnancy-related calls to 108 across the five states during the study period (table 1). The median age of women in this study was 23 years (IQR $21-25)$, with few women $<18(0.01 \%)$ or older than $34(1.8 \%)$. Women were largely from rural or tribal areas $(92.1 \%)$, and overall transport times were significantly longer for tribal and rural areas compared with urban areas $(p<0.0001)$ (table 2$)$. However, only $5.5 \%$ of transports took $>2$ hours, with none lasting longer than 3 hours.

Almost half of all women had attended at least four antenatal care visits, as recommended by the WHO. By self-report, few current or previous pregnancies were complicated by anaemia or hypertension. While almost all women presented with contractions $(96.7 \%)$, only $29.3 \%$ of women had rupture of membranes prior to EMT arrival (table 3). EMTs consistently measured basic 
Table 1 Characteristics of women in their third trimester of pregnancy transported by emergency medical systems for pregnancy-related problem

\begin{tabular}{|c|c|c|c|c|}
\hline Characteristics* & $\begin{array}{l}\text { All } \\
\text { N (\%) }\end{array}$ & $\begin{array}{l}\text { Delivered } \\
\mathbf{N}(\%)\end{array}$ & $\begin{array}{l}\text { Did not deliver } \\
\text { N (\%) }\end{array}$ & $\begin{array}{l}\text { Not followed } \dagger \\
\text { N (\%) }\end{array}$ \\
\hline All patients & 1684 & 1411 & 188 & 85 \\
\hline \multicolumn{5}{|l|}{ Age } \\
\hline Median (IQR) & $23(21-25)$ & $23(21-26)$ & $24(21-26)$ & $23(21-25)$ \\
\hline $15-19$ & $83(4.9)$ & $70(5)$ & $7(3.7)$ & $6(7.1)$ \\
\hline $20-24$ & 958 (56.9) & $797(56.5)$ & $106(56.4)$ & $55(64.7)$ \\
\hline $25-29$ & $500(29.7)$ & 428 (30.3) & $58(30.9)$ & $14(16.5)$ \\
\hline $30-34$ & $112(6.7)$ & $90(6.4)$ & $13(6.9)$ & 9 (10.6) \\
\hline $35-39$ & $27(1.6)$ & $23(1.6)$ & $3(1.6)$ & $1(1.2)$ \\
\hline $40-44$ & $4(0.2)$ & $3(0.2)$ & $1(0.5)$ & $0(0)$ \\
\hline \multicolumn{5}{|l|}{ Geographic location } \\
\hline Rural & $1333(79.2)$ & $1115(79)$ & $153(81.4)$ & $65(76.5)$ \\
\hline Urban & $134(8.0)$ & 107 (7.6) & $13(6.9)$ & $14(16.5)$ \\
\hline Tribal & $217(12.9)$ & $189(13.4)$ & $22(11.7)$ & $6(7.1)$ \\
\hline \multicolumn{5}{|l|}{ Economic status } \\
\hline Pink card & $479(28.4)$ & $415(29.4)$ & $46(24.5)$ & $18(21.2)$ \\
\hline White card & 1177 (69.9\%) & $974(69)$ & $140(74.5)$ & $63(74.1)$ \\
\hline \multicolumn{5}{|l|}{ Social status } \\
\hline Other caste & $343(20.4)$ & 281 (19.9) & 35 (18.6) & $27(31.8)$ \\
\hline Below caste & $608(36.1)$ & $501(35.5)$ & 78 (41.5) & $29(34.1)$ \\
\hline Scheduled caste & $297(17.6)$ & $250(17.7)$ & 35 (18.6) & $12(14.1)$ \\
\hline Scheduled tribe & $430(25.5)$ & $375(26.6)$ & $39(20.7)$ & $16(18.8)$ \\
\hline \multicolumn{5}{|l|}{ Education } \\
\hline None & $637(37.8)$ & $520(36.9)$ & 87 (46.3) & $30(35.3)$ \\
\hline Primary & $429(25.5)$ & $354(25.1)$ & $54(28.7)$ & $21(24.7)$ \\
\hline Secondary & $428(25.4)$ & $376(26.7)$ & $34(18.1)$ & $18(21.2)$ \\
\hline Intermediate & $90(5.3)$ & $81(5.7)$ & $5(2.7)$ & $4(4.7)$ \\
\hline Graduate & $40(2.4)$ & 33 (2.3) & $5(2.7)$ & $2(2.4)$ \\
\hline \multicolumn{5}{|l|}{ Obstetric history } \\
\hline Anaemia & $125(7.4)$ & $97(6.9)$ & $18(9.6)$ & $10(11.8)$ \\
\hline Hypertension & $42(2.5)$ & $39(2.8)$ & $2(1.1)$ & $1(1.2)$ \\
\hline \multicolumn{5}{|l|}{ Antenatal care visits } \\
\hline 0 & $108(6.4)$ & $92(6.5)$ & $14(7.5)$ & $2(2.4)$ \\
\hline 1 & $142(8.4)$ & $117(8.3)$ & $23(12.2)$ & $2(2.4)$ \\
\hline 2 & $235(14.0)$ & $196(13.9)$ & $22(11.7)$ & $17(20)$ \\
\hline 3 & $384(22.8)$ & 317 (22.5) & 38 (20.2) & $29(34.1)$ \\
\hline $4+$ & 778 (46.2) & 661 (46.9) & $87(46.3)$ & $30(35.3)$ \\
\hline Seen by physician during visit & $1309(77.7)$ & 1095 (77.6) & $139(73.9)$ & $75(88.2)$ \\
\hline \multicolumn{5}{|l|}{ Parity } \\
\hline Nulliparous & $725(43.1)$ & $803(56.9)$ & $117(62.2)$ & 39 (45.9) \\
\hline Multiparous & 959 (56.9) & $608(43.1)$ & $71(37.8)$ & $46(54.1)$ \\
\hline \multicolumn{5}{|l|}{ Age at first pregnancy $\ddagger$} \\
\hline $15-19$ & $227(23.7)$ & 192 (23.9) & $29(24.8)$ & $6(15.4)$ \\
\hline $20-24$ & $647(67.5)$ & $541(67.4)$ & $79(67.5)$ & $27(69.2)$ \\
\hline $25-29$ & $76(7.9)$ & $64(8)$ & $8(6.8)$ & $4(10.3)$ \\
\hline $30-34$ & $4(0.4)$ & $3(0.4)$ & $0(0)$ & $1(2.6)$ \\
\hline $35-39$ & $1(0.1)$ & $0(0)$ & $1(0.9)$ & $0(0)$ \\
\hline \multicolumn{5}{|l|}{ Prior caesarean section $\ddagger$} \\
\hline Yes & $89(9.3)$ & $70(8.7)$ & $16(13.7)$ & $3(7.7)$ \\
\hline No & $865(90.2)$ & $728(90.7)$ & 101 (86.3) & $36(92.3)$ \\
\hline \multicolumn{5}{|l|}{ Years since prior pregnancy } \\
\hline$<2$ years & $435(45.4)$ & $358(44.6)$ & $56(47.9)$ & $21(53.9)$ \\
\hline 24-35 months & $240(25.0)$ & 207 (25.8) & $25(21.4)$ & 8 (20.5) \\
\hline$>3$ years & 277 (28.9) & 232 (28.9) & $36(30.8)$ & 9 (23.1) \\
\hline
\end{tabular}

*Values may not add up to $100 \%$ as most categories have missing data. All missing data were $<6 \%$.

†'Not followed' are patients lost to follow-up prior to delivering.

$\ddagger$ Of multiparous mothers only ( $n=959$ ). 
Table 2 Response and transport times for women transported by emergency medical systems for pregnancy-related problems

\begin{tabular}{lcccc}
\hline & & Incident location & & \\
Characteristic & All & Urban & Rural/tribal & P Value \\
\hline Response time $(\mathrm{min})$ & & & & \\
$\quad$ Call to dispatch & $3(2-4)$ & $3(2-4)$ & $3(2-4)$ & 0.55 \\
Dispatch to scene & $24(16-35)$ & $17(11-28)$ & $25(16-35)$ & $<0.0001$ \\
Time on scene & $7(5-10)$ & $9(5-12)$ & $7(5-10)$ & 0.076 \\
Scene to hospital & $26(17-40)$ & $22(12-35)$ & $26(18-40)$ & 0.005 \\
$\quad$ Total time: call to hospital & $65(50-84)$ & $56(42-73)$ & $66(51-84)$ & $<0.0001$ \\
Distance from scene to hospital $(\mathrm{km})$ & $15(9-23)$ & $12(6-17)$ & $15(9-23)$ & $<0.0001$ \\
\hline All values are median (IQR). & & & &
\end{tabular}

vitals, and properly positioned mothers in the left lateral position en route. Twenty-four women presented with signs of severe pre-eclampsia, defined as systolic blood pressure $>160 \mathrm{~mm} \mathrm{Hg}$ or diastolic blood pressure $>110 \mathrm{~mm} \mathrm{Hg}$, or eclampsia, defined by an EMT witnessed seizure. Only one of these women $(4.5 \%)$ received magnesium as indicated by standard GVK EMRI protocol.

Of enrolees, 1411 mothers delivered during the study period; $186(11 \%)$ women did not deliver by 7 days and were excluded from further follow-up; and 87 (5.2\%) women were lost to follow-up prior to delivering. Of these 1411 mothers, 80 delivered in the prehospital setting, of which 36 delivered prior to arrival of the EMT and an additional 44 delivered on scene or during transport to the hospital (table 3). Of these 44 EMT-assisted deliveries, EMTs regularly delivered the placenta and provided transabdominal uterine massage. In only $1.3 \%$ of all prehospital deliveries, including deliveries prior to EMT arrival and EMT assisted, did the EMT administer oxytocin. In that case, it was given to a woman with postpartum haemorrhage $(\mathrm{PPH})$. However, there were no

\begin{tabular}{lc}
$\begin{array}{l}\text { Table } 3 \\
\text { transported by emerentation and EMT management of women } \\
\text { pregnancy-related problems }\end{array}$ \\
\hline Patient presentation and management & $\mathbf{N ~ ( \% )}$ \\
\hline All patients & 1684 \\
Presentation & $1628(96.7 \%)$ \\
$\quad$ Contractions & $493(29.3 \%)$ \\
Rupture of membranes & $22(1.3 \%)$ \\
Severe pre-eclampsia & $2(0.1 \%)$ \\
Eclampsia & \\
EMT actions & $1633(97.0 \%)$ \\
Pulse, blood pressure and respiratory & \\
rate measured & $1610(95.6 \%)$ \\
Placed in left lateral decubitus position & 44 \\
Deliveries assisted by an EMT & \\
Active management of third stage of labour* & \\
Placental delivery & $33(75 \%)$ \\
Oxytocin & $0(0 \%)$ \\
Uterine massage $\dagger$ & $29(87.9 \%)$ \\
*Of EMT-assisted deliveries $(n=44)$. & \\
†Of patients whose placenta was delivered $(n=33)$. & \\
EMT,emergency medical technician. &
\end{tabular}

incidents of documented severe PPH, defined by $1 \mathrm{~L}$ or more of estimated blood loss. In $95 \%$ of the cases where oxytocin was not administered post-delivery, EMTs stated the reason that it was 'not indicated'.

Follow-up rates at 48 hours, 7 days and 42 days were $95.0 \%, 94.4 \%$ and $94.1 \%$, respectively. In total, four women died during this study, and all died within 48 hours after arrival at the hospital. One of these women presented with eclampsia, but the final aetiology of their deaths is unknown. Most women delivered at a hospital $(85.9 \%)$, and those were overwhelmingly at government hospitals $(82.9 \%)$. There were 154 deliveries $(10.9 \%)$ that occurred at home, and $81.8 \%$ of these occurred after being discharged from the hospital to which EMTs had originally transported women. EMTs assisted in 44 deliveries (3.2\%).

The overall rate of caesarean section was $8.2 \%$. Karnataka and Meghalaya each had only one woman deliver by caesarean section; therefore, these states were not included in univariate and multivariate regression analysis predicting caesarean section. State, hospital type (private vs government), hospital level (eg, primary vs tertiary) and prior caesarean section were significantly correlated with caesarean section on univariate analysis.

Multivariate logistic regression analysis identified several factors that significantly impacted the likelihood of caesarean section $(\mathrm{n}=791 ; c$-statistic 0.75$)$ (table 4). Women who were initially transported to a tertiary care centre, such as a medical college, were significantly less likely to deliver by caesarean section than those initially taken to primary care centres (OR 0.16 (95\% CI 0.07 to $0.37)$ ). Similarly, women initially transported to a private hospital rather than a government primary care centre were significantly less likely to deliver by caesarean section $(0.17$ (0.06 to 0.46$))$. In contrast, women with a history of a previous caesarean section or who were nulliparous were more likely to deliver by caesarean section (2.96 (1.71 to 5.10$)$ and 3.36 (1.47 to 7.71$)$, respectively).

\section{DISCUSSION}

This is the first prospective study to evaluate the characteristics and outcomes of obstetric patients transported by the world's largest EMS organisation, GVK EMRI. Our study enrolled patients from one-third of the 
Table 4 Predictors of caesarean section, multivariate analysis

\begin{tabular}{lll}
\hline Characteristics & OR (95\% Cl) & Adjusted \\
\hline State & Unadjusted & Ref \\
$\quad$ Gujarat & Ref & $1.86(0.98$ to 3.54$)$ \\
Andhra Pradesh & $1.84(1.1$ to 3.08$)$ & $3.22(1.70$ to -6.10$)$ \\
Assam & $3.98(2.34$ to 6.77$)$ & $1.11(1.04$ to 1.19$)$ \\
Age & $1.04(0.98$ to 1.09$)$ & $1.19(0.63$ to 2.26$)$ \\
Low economic status & $0.84(0.5$ to 1.4$)$ & Ref \\
Receiving hospital type & & $0.48(0.29$ to 0.78$)$ \\
Primary, government & Ref & $0.16(0.07$ to 0.37$)$ \\
$\quad$ Secondary, government & $0.62(0.39$ to 0.97$)$ & $0.17(0.06$ to 0.46$)$ \\
$\quad$ Tertiary, government & $0.21(0.1$ to 0.45$)$ & $0.22(0.06$ to 0.77$)$ \\
Private & $0.17(0.07$ to 0.43$)$ & \\
$\quad$ Other & $0.19(0.06$ to 0.62$)$ & \\
Caesarean section history & & \\
Multiparous, no prior & Ref & $3.36(1.47$ to 7.71$)$ \\
$\quad$ Nulliparous & $2.06(0.97$ to 4.38$)$ & $2.96(1.71$ to 5.10$)$ \\
Multiparous, prior caesarean section & $1.61(1.06$ to 2.44$)$ & $3.51(0.96$ to 12.75$)$ \\
$\quad$ Twin gestation & $2.69(0.83$ to 8.73$)$ & $2.15(1.19$ to 3.89$)$ \\
\hline Premature gestation & $1.62(0.95$ to 2.76$)$ &
\end{tabular}

states in which GVK EMRI operates, providing evidence of the vast potential of a centralised EMS to reach vulnerable women during the third trimester and childbirth.

\section{Providing timely care for vulnerable populations}

Leveraging existing EMS resources, such as dispatch centre, ambulances and care providers, increases the capacity to reach vulnerable women during childbirth and decreases time to facility-based obstetric care. Our findings demonstrate that women from vulnerable populations were able to access emergency obstetric services by phone, either directly or through a friend or relative. Of the women transported, $<40 \%$ had a secondary level education and $70 \%$ were dependent on the low-income government health insurance programme (white ration card). Using self-identified caste as a proxy of social status, we also found that almost $80 \%$ of patients were from lower social strata. These categories are used as they are in national population health level monitoring: 'scheduled caste' is considered the lowest, most socially disadvantaged group, whereas 'scheduled tribe', also a disadvantaged group, is defined by their physical isolation; 'below caste' is an intermediary group socially; and 'other caste' includes all those who do not belong to the aforementioned group and have the highest social status. Moreover, with a median call-to-facility arrival time of $65 \mathrm{~min}$ (IQR 50-84), this overwhelmingly rural population was connected quickly to facility-base care. This is in accordance with the WHO and the United Nations Population Fund (UNFPA) recommendations that labouring women have access to emergency obstetric and newborn care (EmONC) facilities within 2 hours. Longer times have been associated with worse outcomes including higher maternal mortality. ${ }^{16}{ }^{17}$ However, one population was not well represented in our sample: women $<18$ years old. Only one patient $(0.01 \%)$ enrolled in our study was $<18$ years. This is much lower than expected, given that $2.5 \%$ of all women enrolled, and $22 \%$ of women nationally report having their first pregnancy before age $18 .^{18}$ The reason for this unexpectedly low percentage may be that women $<18$ years have restricted autonomy and/or lower health literacy than older women. Further investigation is needed.

\section{Providing appropriate care for prehospital deliveries}

In advance of assessing and treating pregnant patients, over $99 \%$ of GVK EMRI's EMTs have undergone Basic Life Support in Obstetrics (BLSO) training in addition to their initial EMT B training (ranging from 6 weeks previously to 10 weeks ( 450 hours) currently). Appropriate practices such as obtaining maternal vital signs and placing the patient in the left lateral decubitus position were performed in almost all patients. Of our study patients, EMTs assisted in the delivery of $44(3.1 \%)$ patients. Of these, the placenta was delivered in $75 \%$ of patients and most patients received transabdominal uterine massage. Active management of the third stage of labour (AMSTL) is within GVK EMRI's EMTs' scope of practice and is highlighted in their emergency care protocols. Despite this, not a single patient received oxytocin, the key component of AMSTL, following an EMT-assisted delivery. ${ }^{19}$ In fact, for the cohort of prehospital deliveries, $95 \%$ of EMTs reported that administration was not indicated. The rationale for this misconception, despite access to standard protocols and contact with call centre physicians for real-time medical direction, is likely multifactorial. Possible explanations 
include a lack of provider comfort with oxytocin administration and the overall protocol, or an environment where physicians may not be supportive of EMTs providing oxytocin. Further, there are additional opportunities to improve the quality of EmONC beyond AMSTL. Only one patient with PPH received oxytocin, and no patients with eclampsia or severe preeclampsia received magnesium. GVK EMRI has already begun responding to these quality gaps by conducting EmONC refresher programmes for practicing EMTs. Further focused efforts at the institutional, development partner and government levels will likely be needed. Potential solutions include multi-agency, multispecialty quality improvement efforts that bring together key stakeholders from healthcare facilities, government and prehospital providers. Together, these groups can collectively solve the problem and elucidate regional standards of care, including scope of practice for EMTs, continuing medical education and standardised certification.

\section{Improving facility-based deliveries}

In this study, women recognised the appropriate time frame to come to a facility for a delivery. The vast majority of women $(93 \%)$ delivered within $\sim 48$ hours of the original dispatch call. A significant number of patients transported to hospitals who were subsequently discharged, delivered within the next 2 days at home, constituting $7.2 \%$ of all deliveries. Perhaps most striking is that $82 \%$ of these postdischarge home deliveries occurred at the day of transport. This may be an opportunity for facility-based quality improvement regarding the detection of early labour and patient discharge education, or system-based interventions such as maternity waiting homes. ${ }^{20}$

The overall caesarean section rate of $8.2 \%$ was below the national average of $12.1 \%$ in India ${ }^{21}$ and the traditionally recommended rate by the $\mathrm{WHO}$ of $10-15 \% .^{22}$ Prior reports have suggested that delivery in private facilities is associated with increased rates of caesarean delivery in India and other South Asian countries. ${ }^{23}$ However, this did not hold true in rural India, where public facilities were found to have higher rates of caesarean delivery. ${ }^{24}$ Our study is consistent with this latter finding. Caesarean section rates in private hospitals were $3.5 \%$ compared with $10.9 \%$ in public hospitals, with the highest rate in rural public hospitals (11.2\%). Further, the increased likelihood of delivering by caesarean section if transported to a primary care centre may be indicative of multiple different clinical scenarios. Future studies should examine interfacility transfers for obstetric emergencies to determine the need for obstetric emergency-specific referral protocols.

\section{Limitations}

Any conclusions regarding maternal mortality are limited as there were few maternal deaths in our sample. The estimated MMR for our study population is at least 280, but may be as high as 291, if all infants that died the day of birth are assumed to be stillborn. Without taking into account the known deep disparity between urban and rural MMR's, ${ }^{10}$ the expected MMR would be $152,{ }^{25}$ weighted by a state's proportion of our sample size but not inclusive of Meghalaya, which has no available recent MMR. The generalisability of our findings is limited by a lack of data collection beyond daytime hours and the predominance of three of the five states in our sample. Lastly, two factors may limit the accuracy of our caesarean section rates: patients lost to follow-up and missing data. While our follow-up rates were strong, we still lost 168 patients in follow-up and for 139 women we did not have the mode of delivery recorded. These women may have had different rates of caesarean section and/or complications, including death.

\section{CONCLUSIONS}

Pregnant women from vulnerable Indian populationsgeographically isolated, low socioeconomic status-use a free-of-charge ambulance service for impending delivery. EMTs regularly deliver women in the field and consistently perform basic assessment and management of pregnant patients, reaching women within the internationally recommended 2 hours of EmONC. Together, the ability to reach vulnerable populations, provide care and connect women makes EMS an integral part of the healthcare system. We have identified several areas in need of quality improvement including AMSTL and the management of $\mathrm{PPH}$, eclampsia and severe preeclampsia. Future research and health system planning should focus on how to strengthen and expand EMS as a critical component of emergency obstetric care services.

\section{Author affiliations}

${ }^{1}$ Department of Emergency Medicine, Stanford University, Stanford, California, USA

${ }^{2}$ Department of Emergency Medicine, University of California San Francisco, San Francisco, California, USA

${ }^{3}$ Department of Surgery, Division of Plastic Surgery, University of Washington/Harborview Medical Center, Seattle, Washington, USA ${ }^{4}$ Department of Family Medicine and Community Health, University of Wisconsin School of Medicine and Public Health, Verona, Wisconsin, USA ${ }^{5}$ Departments Family and Community Medicine and Obstetrics \& Gynecology, University of New Mexico, Albuquerque, New Mexico, USA

${ }^{6}$ GVK Emergency Management and Research Institute, Secunderabad, Telangana, India

Acknowledgements The authors give their special thanks to Anne Tecklenberg Strehlow for her assistance in editing and Aruna Gimkala, Marada Lakshmana Rao, Royal Uddin Ahmed, Rupjoy Maibangsa, Rajini Danthala, Divya Patel, Steffy Christian, Chandrashekhraswami Kendadmath, Sahyadri Venkateshappa, Shylaja Muniyappa and Isberth Tham for collecting data and ensuring data quality.

Contributors MCS, EAP, GVRR and SVM contributed to the study design, implementation, data analysis and manuscript production. HEM, AEE and LL contributed to study design and manuscript production. JAN and CBB contributed to data analysis and manuscript production. MCS and SVM accept full responsibility for the work and conduct of the study, had access to the data and controlled the decision to publish. MCS affirms that this manuscript is an honest, accurate and transparent account of the study being reported; that no important aspects of the study have been omitted; and that any discrepancies from the study as planned (and, if relevant, registered) have been explained. 
Competing interests None declared.

Ethics approval Stanford University IRB and GVK EMRI Ethics Review Board.

Provenance and peer review Not commissioned; externally peer reviewed.

Data sharing statement Extra data can be accessed via the Dryad data repository at http://datadryad.org/ with the doi:10.5061/dryad.g08gb. Full data set available upon request from the corresponding author at strehlow@stanford. edu. Consent was not obtained but the presented data are anonymised and risk of identification is low.

Open Access This is an Open Access article distributed in accordance with the Creative Commons Attribution Non Commercial (CC BY-NC 4.0) license, which permits others to distribute, remix, adapt, build upon this work noncommercially, and license their derivative works on different terms, provided the original work is properly cited and the use is non-commercial. See: http:// creativecommons.org/licenses/by-nc/4.0/

\section{REFERENCES}

1. United Nations. The Millennium Development Goals Report 2015. 2015. http://www.un.org/millenniumgoals/2015 MDG_Report/pdf/ MDG 2015 rev (July 1).pdf

2. U. N. General Assembly, 70th Session. Resolution A/RES/70/1 (2015) [Transforming our world: The 2030 Agenda for Sustainable Development]. 2015 (cited 12 January 2016). http://www.un.org/en/ ga/70/resolutions.shtml

3. Temmerman M, Khosla R, Bhutta ZA, et al. Towards a new global strategy for women's, children's and adolescents' health. BMJ 2015;351:h4414.

4. World Health Organization. Global Strategy for Women's, Children's and Adolescences Health. 2015. http://who.int/life-course/partners/ global-strategy/globalstrategyreport2016-2030-lowres.pdf

5. Okong P, Byamugisha J, Mirembe F, et al. Audit of severe maternal morbidity in Uganda-implications for quality of obstetric care. Acta Obstet Gynecol Scand 2006;85:797-804.

6. Cham M, Vangen S, Sundby J. Maternal deaths in rural Gambia Glob Public Health 2007;2:359-72.

7. Hirose A, Borchert M, Cox J, et al. Determinants of delays in travelling to an emergency obstetric care facility in Herat, Afghanistan: an analysis of cross-sectional data and spatial modelling. BMC Pregnancy Childbirth 2015;15:14

8. Hussein J, Kanguru L, Astin M, et al. The effectiveness of emergency obstetric referral interventions in developing country settings: a systematic review. PLoS Med 2012;9: e1001264.

9. World Health Organization. Trends in Maternal Mortality: 1990 to 2015 Estimates by the WHO, UNICEF, UNFPA, World Bank Group and the United Nations Population Division. 2015. http://www.who.int/ reproductivehealth/publications/monitoring/maternal-mortality-2015/ en/
10. Montgomery AL, Ram U, Kumar R, et al. Maternal mortality in India: causes and healthcare service use based on a nationally representative survey. PLOS ONE 2014;9:e83331.

11. Sidney K, Ryan K, Diwan V, et al. Utilization of a state run public private emergency transportation service exclusively for childbirth: the Janani (maternal) Express program in Madhya Pradesh, India. PLOS ONE 2014;9:e96287.

12. GVK Emergency Management Research Institute. National Daily Report. 2014. [Internal Report]. 2014.

13. McLelland GE, Morgans AE, McKenna LG. Involvement of emergency medical services at unplanned births before arrival to hospital: a structured review. Emerg Med J 2014;31:345-50.

14. Verdile VP, Tutsock G, Paris PM, et al. Out-of-hospital deliveries: a five-year experience. Prehosp Disaster Med 1995;10:10-13.

15. McLelland G, Morgans A, McKenna L. Victorian paramedics' encounters and management of women in labour: an epidemiological study. BMC Pregnancy Childbirth 2015;15:13.

16. Gething PW, Johnson FA, Frempong-Ainguah F, et al. Geographica access to care at birth in Ghana: a barrier to safe motherhood. BMC Public Health 2012;12:991.

17. World Health Organization, UNFPA, UNICEF and Mailman School of Public Health. Monitoring emergency obstetric care. A handbook 2015. http://www.who.int/reproductivehealth/publications/monitoring/ 9789241547734/en

18. Moore AM, Singh S, Ram U, et al. Adolescent marriage and childbearing in India: current situation and recent trends. New York: Guttmacher Institute, 2009.

19. Begley CM, Gyte GM, Devane D, et al. Active versus expectant management for women in the third stage of labour. Cochrane Database Syst Rev 2015;(3):CD007412.

20. van Lonkhuijzen L, Stekelenburg J, van Roosmalen J. Maternity waiting facilities for improving maternal and neonatal outcome in low-resource countries. Cochrane Database Syst Rev 2012;10: CD006759.

21. Government of India. Ministry of Health \& Family Welfare. Health Management Information System. Performance of Key HMIS Indicators April 2014 to March 2015, All India [Internet]. 2016. (cited 12 January 2016). https://nrhm-mis.nic.in/hmisreports/frmstandard_ reports.aspx

22. World Health Organization, HRP. WHO statement on caesarean section rates [Internet]. 2015. http://apps.who.int/iris/bitstream/ 10665/161442/1/WHO RHR 15.02 eng.pdf?ua=1

23. Randive B, Diwan V, De Costa A. India's Conditional Cash Transfer Programme (the JSY) to promote institutional birth: is there an association between institutional birth proportion and maternal mortality? PLOS ONE 2013;8:e67452.

24. Neuman M, Alcock G, Azad K, et al. Prevalence and determinants of caesarean section in private and public health facilities in underserved South Asian communities: cross-sectional analysis of data from Bangladesh, India and Nepal. BMJ Open 2014;4:e005982.

25. Government of India. Ministry of Home Affairs. Census of India: Sample Registration System 2011-2013 [Internet]. 2016. (cited 12 January 2016). http://www.censusindia.gov.in/2011-Common/ Sample_Registration_System.html 